\title{
Parámetros hematológicos de reproductores de Brycon amazonicus (Bryconidae) en cultivo
}

\author{
Hematological parameters of Brycon amazonicus (Bryconidae) \\ broodstock in captivity \\ Anaí Gonzales ${ }^{1,2,4}$, Giana Curto ${ }^{1,3}$, Christian Fernández-Mendez ${ }^{1}$
}

\section{Resumen}

El objetivo de este estudio fue determinar los valores hematológicos de reproductores de Brycon amazonicus bajo cultivo en Iquitos, Perú. Se tomaron muestras de sangre de 12 ejemplares $(1.62 \pm 0.45 \mathrm{~kg}$ y $51.38 \pm 2.93 \mathrm{~cm})$ mantenidos en estanques de tierra y alimentados dos veces al día con una dieta comercial extruida al $22 \%$ de proteína bruta. Los peces fueron colectados con una red y anestesiados con eugenol. La extracción de sangre se realizó con jeringas descartables conteniendo EDTA (10\%). Los valores obtenidos de la serie eritrocítica fueron: hematocrito $53.3 \pm 4.8 \%$; hemoglobina $20.1 \pm 3.2 \mathrm{~g} / \mathrm{dl}$; eritrocitos $2.8 \pm 0.5 \times 10^{6} / \mu \mathrm{l} ; \mathrm{VCM} 194.4 \pm 54.4 \mathrm{fl} ; \mathrm{CHCM} 37.7 \pm 4.8 \mathrm{~g} / \mathrm{dl} ; \mathrm{HCM} 72.6 \pm 17.7$ pg. El número de leucocitos fue de $8.1 \pm 6.0 \times 10^{3} / \mu 1$. En el recuento diferencial de leucocitos, los linfocitos y monocitos fueron las células de mayor proporción en comparación con los neutrófilos, eosinófilos y basófilos. La proteína total sérica fue de $3.7 \pm 0.4 \mathrm{~g} / \mathrm{dl}$ y la glucosa de $60.2 \pm 39.1 \mathrm{mg} / \mathrm{dl}$. Los valores encontrados para $B$. amazonicus fueron comparados con otras especies del género Brycon mostrando algunas variaciones, pero dentro del rango reportado para peces teleósteos.

Palabras clave: Amazonía; acuicultura; hematología; sanidad

\section{AbStract}

The objective of this study was to determine the hematological values of Brycon amazonicus broodstock under culture in Iquitos, Peru. Blood samples were taken from 12 specimens $(1.62 \pm 0.45 \mathrm{~kg}$ and $51.38 \pm 2.93 \mathrm{~cm})$ maintained in earthen ponds and fed twice

\footnotetext{
${ }^{1}$ Instituto de Investigaciones de la Amazonía Peruana-IIAP, Programa para el Uso y Conservación del Agua y sus Recursos-AQUAREC, Iquitos, Perú

${ }^{2}$ Programa de Pós-Graduação em Biodiversidade Tropical (PPGBIO), Universidade Federal do Amapá (UNIFAP), Macapá, Brasil

${ }^{3}$ Dirección Regional de la Producción de Loreto, Gobierno Regional de Loreto, Iquitos, Loreto, Perú

${ }^{4}$ E-mail: anaigonzalesf@gmail.com
}

Recibido: 25 de julio de 2018

Aceptado para publicación: 2 de enero de 2019 
a day with a commercial diet with $22 \%$ crude protein. The fish were collected with a net and anesthetized with eugenol. Blood extraction was performed with disposable syringes containing EDTA $(10 \%)$. The values obtained from the erythrocytic series were: hematocrit $53.3 \pm 4.8 \%$; hemoglobin $20.1 \pm 3.2 \mathrm{~g} / \mathrm{dl}$; erythrocytes $2.8 \pm 0.5 \times 10^{6} / \mu 1$; VCM $194.4 \pm 54.4 \mathrm{fl} ;$ CHCM $37.7 \pm 4.8 \mathrm{~g} / \mathrm{dl} ; \mathrm{HCM} 72.6 \pm 17.7 \mathrm{pg}$. The number of leukocytes was $8.1 \pm 6.0 \times 10^{3} / \mu 1$. In the differential count of leukocytes, the lymphocytes and monocytes had greater proportion in comparison with neutrophils, eosinophils and basophils. The total serum protein was $3.7 \pm 0.4 \mathrm{~g} / \mathrm{dl}$ and the glucose was $60.2 \pm 39.1 \mathrm{mg} / \mathrm{dl}$. The values found for $B$. amazonicus were compared with other species of the genus Brycon showing some variations, but within the range reported for teleost fishes.

Key words: Amazonia; aquaculture; hematology; health

\section{INTRODUCCIÓN}

Brycon amazonicus Spix y Agassiz, 1829 , es un pez nativo de la cuenca del Amazonas (Lima, 2017), presenta características migratorias y régimen alimenticio omnívoro (Blanco-Parra et al., 2006). La alimentación en el medio natural está relacionada con el ciclo hidrobiológico del agua, siendo que en la época de creciente del cauce de los ríos se alimentan de insectos adultos y restos de peces y en la época de vaciante se alimentan de vegetales (Val y Almeida-Val, 1995; Pizango-Paima et al., 2001). La carne de esta especie es apreciada por parte de los pobladores amazónicos y, por lo tanto, es una de las especies de mayor desembarque en los mercados de Iquitos, Perú, superadas solo por especies como Arapaima gigas, Colossoma macropomum y Brachyplatystoma rousseauxii (García-Vásquez et al., 2012).

Actualmente se viene realizando el cultivo de esta especie con potencial piscícola a fin de reducir la presión de pesca, además que posee características favorables como la reproducción por inducción hormonal, aceptación al alimento balanceado y rápido crecimiento (Romagosa et al., 2001; Zaniboni et al., 2006; Nuñez, 2009). Sin embargo, exis- ten factores que limitan el desarrollo de la crianza como es el intenso canibalismo en los primeros estadios de vida (Zaniboni et al., 2006; Leonardo et al., 2008). Se han desarrollado estudios de la respuesta fisiológica del estrés ante la densidad, manipulación y transporte (Carneiro y Urbinati, 2001, 2002; Urbinati et al., 2004; Rocha et al., 2004).

Los parámetros hematológicos son una herramienta útil para medir el estado fisiológico de los peces (Ranzani-Paiva et al., 2013) frente a diversas condiciones; entre ellas, la respuesta al estrés, enfermedades y desnutrición, que pueden alterar el patrón característico del eritrograma y de los valores bioquímicos (Tavares-Días y Moraes, 2004). Es poco lo que se conoce sobre los parámetros hematológicos de B. amazonicus, tanto en el medio natural como en cautiverio. Por lo tanto, se requiere investigar en esta área porque se ha encontrado variaciones dentro de especies del género Brycon, pudiendo deberse a la localidad de distribución o la especie estudiada (Tavares-Días et al., 1999; Arias et al., 2003; Oliveira et al., 2015). De esta manera, el objetivo del presente estudio fue determinar los parámetros hematológicos de reproductores $B$. amazonicus mantenidos en condiciones de cautiverio. 


\section{Materiales y Métodos}

\section{Condiciones de Cultivo}

Se utilizaron 12 ejemplares adultos de B. amazonicus (peso: $1.62 \pm 0.45 \mathrm{~kg}$; longitud total: $51.38 \pm 2.93 \mathrm{~cm}$ ), cultivados en un estanque de tierra, a una densidad de $1 \mathrm{pez} /$ $26 \mathrm{~m}^{2}$, en las instalaciones del Centro de Investigaciones Fernando Alcántara Bocanegra (CIFAB) del Instituto de Investigaciones de la Amazonía Peruana (IIAP), localizado en la carretera Iquitos-Nauta, provincia de Maynas, Loreto, Perú (03 48' 48.9' S - $73^{\circ}$ 19' 2'" O).

Los peces fueron alimentados con una dieta comercial extruido al $22 \%$ de proteína bruta, dos veces al día (08:00 y 14:00) con una tasa de alimentación de $1.5 \%$. Los parámetros de agua se analizaron tres veces al día (07:00, 12:00, 18:00) con mediciones diarias de temperatura $\left(29.31 \pm 1.44{ }^{\circ} \mathrm{C}\right)$, oxígeno disuelto $(6.33 \pm 1.48 \mathrm{mg} / \mathrm{l}), \mathrm{pH}(5.96$ $\pm 0.38)$ y conductividad $(14.70 \pm 1.68 \mu \mathrm{S} / \mathrm{cm})$ con un multiparámetro portátil (Hach, HQ40D). Los parámetros del agua fueron similares a los reportados durante el cultivo de reproductores de $B$. amazonicus (Arias et al., 2003).

\section{Colecta de Sangre y Análisis}

Los peces fueron colectados con una red y anestesiados inmediatamente con eugenol $(0.05 \mathrm{ml} / \mathrm{l})$. Los peces, una vez inmóviles, fueron trasladados con una «almohadilla» a una mesa. Se emplearon toallas húmedas para la inmovilización del pez y vendaje de los ojos. Se extrajo $1 \mathrm{ml}$ de sangre mediante punción de la arteria caudal a nivel del arco hemal, utilizando jeringas desechables de $3 \mathrm{ml}$ conteniendo EDTA (10\%). Una parte de cada muestra se colocó en tubos de $2 \mathrm{ml}$ y fueron conservadas en refrigeración $\left(4{ }^{\circ} \mathrm{C}\right)$ para preservar la viabilidad de las células, mientras que la otra parte se colocó en tubos de $0.5 \mathrm{ml}$ y centrifugadas para obtener el plasma sanguíneo con el fin de realizar las pruebas bioquímicas.

Se realizó la determinación de hematocrito (Hct) por el método del microhematocrito (Goldenfarb et al., 1971), concentración de hemoglobina $(\mathrm{Hb})$ por el método de la cianometahemoglobina (Laboratorios BIOLAB, UK) a $540 \mathrm{~nm}$, conteo de eritrocitos (Er) y leucocitos (Leu) con la solución Natt-Herrick (Natt y Herrick, 1952) a través de la cámara de Neubauer. La determinación de los índices hematimétricos: volumen corpuscular medio (VCM), concentración de hemoglobina corpuscular media (CHCM) y hemoglobina corpuscular media (HCM) según el método de Wintrobe (1934). Para el recuento diferencial de leucocitos se realizaron frotis sanguíneos teñidos con reactivo Wright por $10 \mathrm{~min}$ (Hawkey y Dennett, 1989). Para los parámetros bioquímicos, se determinó la concentración de proteínas totales (método colorimétrico de Biuret: Proti2: Laboratorios Wiener ${ }^{\circledR}$ ) a 540 nm y glucosa (método Trinder: Laboratorios FAR Diagnostics) a $510 \mathrm{~nm}$. Las lecturas fueron realizadas en un espectofotómetro UV visible (Agilent Technologies, Cary 60. California, EEUU).

\section{Análisis de Datos}

Los datos se analizaron mediante estadística descriptiva, considerando promedio, desviación estándar, valor mínimo y máximo. Para determinar la correlación entre las variables se usó el coeficiente de Pearson (r), con el software estadístico «Sigma Plot 11».

\section{Resultados}

Los resultados de la serie eritrocítica y bioquímica sérica con mayor variación fueron el HCM, VCM y glucosa (Cuadro 1). De la serie leucocitica, los linfocitos fueron las células más abundantes, seguidas por los monocitos, mientras que los neutrofilos, basófilos y eosinofilos fueron las células menos frecuentes (Cuadro 2). 
Cuadro 1. Valores de la serie eritrocítica y bioquímica sérica de reproductores de Brycon amazonicus $(\mathrm{n}=12)$ cultivados en Iquitos, Perú

\begin{tabular}{lcc}
\hline Parámetros & Promedio $\pm \mathrm{DE}$ & Mínimo - Máximo \\
\hline Hematocrito $(\%)$ & $53.4 \pm 4.9$ & $47.4-62.6$ \\
Hemoglobina $(\mathrm{g} / \mathrm{dl})$ & $20.1 \pm 3.3$ & $17.0-29.0$ \\
Eritrocitos $\left(10^{6} / \mu \mathrm{l}\right)$ & $2.9 \pm 0.6$ & $1.9-4.0$ \\
VCM $(\mathrm{fl})$ & $194.5 \pm 54.5$ & $137.2-307.2$ \\
CHCM $(\mathrm{g} / \mathrm{dl})$ & $37.8 \pm 4.9$ & $31.3-49.3$ \\
HCM $(\mathrm{pg})$ & $72.6 \pm 17.8$ & $46.0-104.5$ \\
Proteína $(\mathrm{g} / \mathrm{dl})$ & $3.7 \pm 0.4$ & $3.0-4.3$ \\
Glucosa $(\mathrm{mg} / \mathrm{dl})$ & $60.2 \pm 39.1$ & $14.7-115.3$ \\
\hline
\end{tabular}

$\mathrm{VCM}=$ volumen corpuscular medio; $\mathrm{HCM}$ = concentración de hemoglobina; $\mathrm{CHCM}=$ concentración de hemoglobina corpuscular media. DE: desviación estándar

Cuadro 2. Valores de la serie leucocítica absoluta y relativa de reproductores de Brycon amazonicus $(\mathrm{n}=12)$ cultivados en Iquitos, Perú

\begin{tabular}{lcc}
\hline Parámetros & Promedio $\pm \mathrm{DE}$ & Mínimo - Máximo \\
\hline Valores absolutos & & \\
Leucocitos $\left(10^{3} / \mu 1\right)$ & $6.5 \pm 2.4$ & $3.6-12.0$ \\
Linfocitos $\left(10^{3} / \mu 1\right)$ & $5.2 \pm 2.2$ & $2.6-9.8$ \\
Monocitos $\left(10^{3} / \mu 1\right)$ & $1.1 \pm 0.8$ & $0.0-2.9$ \\
Basófilos $\left(10^{3} / \mu 1\right)$ & $0.2 \pm 0.4$ & $0.0-1.4$ \\
Eosinófilos $\left(10^{3} / \mu 1\right)$ & $0.08 \pm 0.19$ & $0.0-0.5$ \\
Neutrófilos $\left(10^{3} / \mu 1\right)$ & $0.02 \pm 0.05$ & $0.0-0.15$ \\
Valores relativos & & \\
Linfocitos & $79.5 \pm 9.9$ & $66.0-96.0$ \\
Monocitos $(\%)$ & $16.5 \pm 9.7$ & $0.0-34.0$ \\
Basófilos $(\%)$ & $2.0 \pm 3.7$ & $0.0-10.0$ \\
Eosinófilos $(\%)$ & $1.5 \pm 4.1$ & $0.0-14.0$ \\
Neutrófilos $(\%)$ & $0.5 \pm 1.2$ & $0.0-4.0$ \\
\hline
\end{tabular}

DE: desviación estándar

Se encontró correlación positiva $(\mathrm{p}<0.05)$ lineal entre VCM-hematocrito, HCM-hematocrito, CHCM-hemoglobina y VCM-HCM, y lineal negativa entre HCMeritrocitos, VCM-eritrocitos, mostrando un buen coeficiente de correlación $(r>0.5)$ (Figura 1).

\section{Discusión}

Al comparar los valores de los parámetros eritrocíticos con otras especies de reproductores de Brycon (Cuadro 3), se aprecia que los valores de eritrocitos y de 

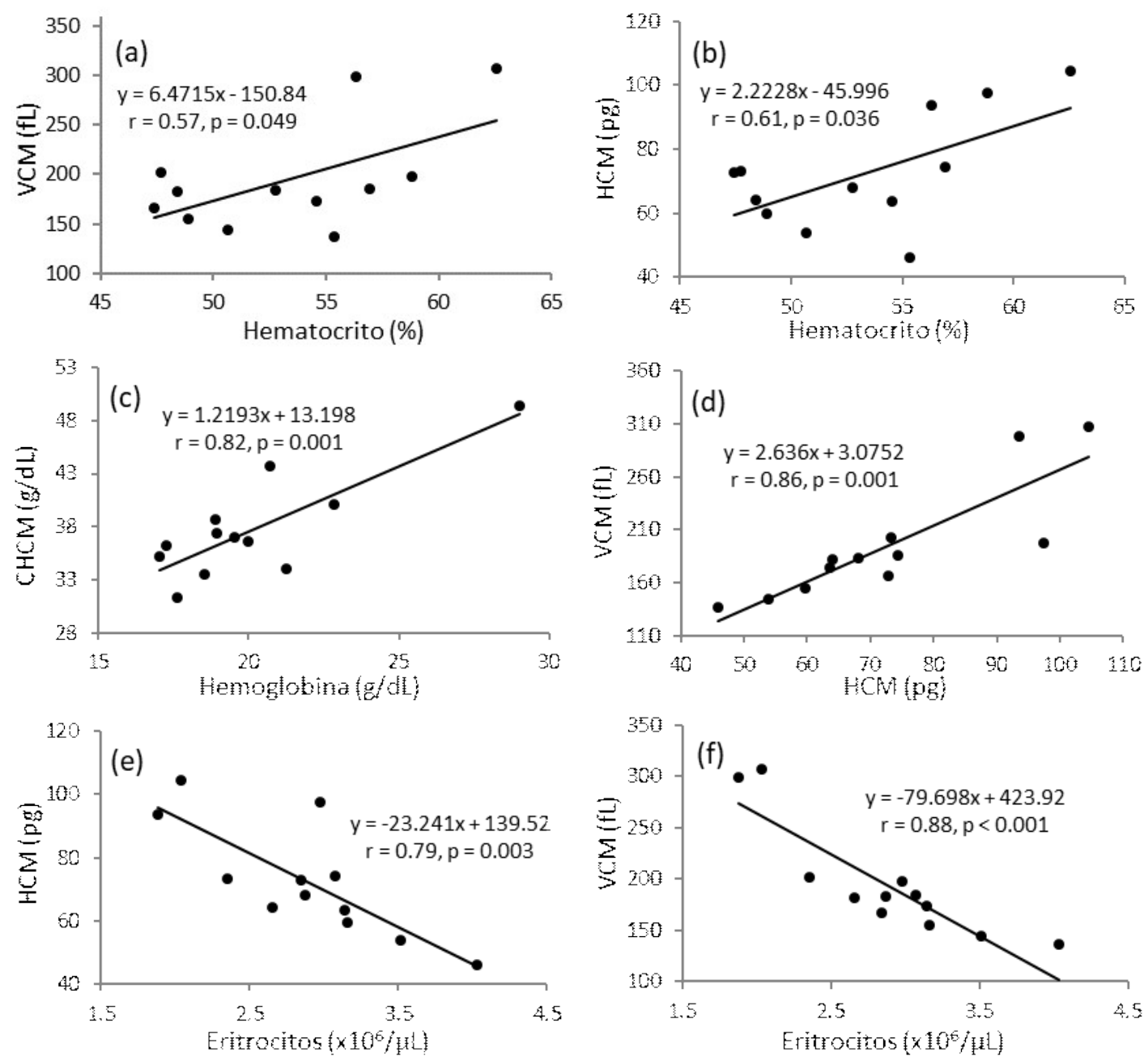

Figura 1. Correlación lineal positiva en (a) VCM-hematocrito, (b) HCM-hematocrito, (c) CHCMhemoglobina, (d) VCM-HCM y negativa en (e) HCM-eritrocitos, (f) VCM-eritrocitos en reproductores de Brycon amazonicus en cautiverio

VCM son similares para B. amazonicus. Sin embargo, los valores de hematocrito, hemoglobina, HCM y CHCM variaron ampliamente y superaron los promedios reportados para reproductores de $B$. insignis y $B$. amazonicus (Ranzani-Paiva, 1991; Arias et al., 2003). No obstante, al ser comparados con valores de alevinos y juveniles de $B$. amazonicus y $B$. orbignyanus mantenidos en cautiverio mostraron el mismo patrón de comportamiento (Arias et al., 2003; Tavares y Moraes, 2006; Tavares-Días et al., 1999, 2008), y coincide con valores reportados en otros teleósteos (Tavares-Días y Moraes, 2004). Tales variaciones de estos parámetros hematológicos pueden ser atribuidas a factores intra e interespecíficos como el sexo, edad, maduración gonadal, variación genética, hábitat, hábito alimenticio, clima y estrés causado durante la manipulación (Tandon y Joshi, 1976; Tavares-Días et al., 2003; TavaresDías y Moraes, 2004). 
Cuadro 3. Comparación de los valores promedio y desviación estándar de la serie eritrocítica de reproductores en cautiverio de Brycon

\begin{tabular}{lcccc}
\hline Parámetros & $\begin{array}{c}\text { Brycon insignis } \\
\text { (Ranzani- } \\
\text { Paiva, 1991) }\end{array}$ & $\begin{array}{c}\text { Brycon } \\
\text { insignis } \\
\text { (Ranzani- } \\
\text { Paiva, 1991) }\end{array}$ & $\begin{array}{c}\text { Brycon } \\
\text { amazonicus } \\
\text { (Arias } \text { et al., } \\
\text { 2003) }\end{array}$ & $\begin{array}{c}\text { Brycon } \\
\text { amazonicus } \\
\text { Presente } \\
\text { estudio }\end{array}$ \\
\hline Hematocrito (\%) & $42.4 \pm 0.5$ & $33.5 \pm 0.7$ & $42.4 \pm 5.6$ & $53.4 \pm 4.9$ \\
Hemoglobina (g/dl) & $10.6 \pm 0.1$ & $8.0 \pm 0.2$ & $14.5 \pm 1.8$ & $20.1 \pm 3.3$ \\
Eritrocitos $\left(10^{6 / \mu l)}\right.$ & $2.08 \pm 0.03$ & $1.78 \pm 0.03$ & $2.8 \pm 0.9$ & $2.9 \pm 0.6$ \\
VCM (fl) & $207.0 \pm 2.2$ & $186.5 \pm 2.9$ & $171.9 \pm 73.7$ & $194.5 \pm 54.5$ \\
CHCM (g/dl) & $25.2 \pm 0.2$ & $24.2 \pm 0.3$ & $34.9 \pm 4.6$ & $37.8 \pm 4.9$ \\
HCM $(\mathrm{pg})$ & $51.6 \pm 0.5$ & $44.4 \pm 0.7$ & $59.8 \pm 26.7$ & $72.6 \pm 17.8$ \\
\hline
\end{tabular}

Mayores valores de hematocrito se presentan en peces más activos y menores en peces de ambientes lénticos y sedentarios (Larsson et al., 1976). Sin embargo, otros factores externos también pueden interferir en el porcentaje de hematocrito; entre ellos, los anestésicos mal administrados (Coffigny y Perez, 2003), pudiendo actuar como un factor estresante adicional (Carneiro et al., 2002), ocasionando hemoconcentración en peces de agua dulce (McDonald y Milligan, 1997). Así mismo, cambios en el hematocrito pueden deberse a variaciones en el volumen de los eritrocitos (VCM) por factores ambientales y fisiológicos, como el equilibrio ácido-base de la sangre, ocurriendo en muchos peces amazónicos, en donde se evidenciaron cambios en el hematocrito sin comprometer los valores de glóbulos rojos presentes en la sangre (Nikinmaa, 1990; Val y Almeida-Val, 1995). Este mismo comportamiento se observó al comparar con reproductores de B. amazonicus donde se aprecia valores de eritrocitos similares, pero valores superiores de hematocrito y VCM (Arias et al., 2003).

La hemoglobina mostró valores altos, posiblemente debido al estrés ocasionado durante la manipulación y la administración del anestésico (Urbinati y Carneiro, 2001). Así mismo, los valores de HCM y CHCM se incrementaron al ser dependientes de la hemoglobina, mostrando mayor similitud a los valores obtenidos en reproductores de $B$. amazonicus (Arias et al., 2003). En este sentido, todos estos factores expresan la importancia de los estudios hematológicos, principalmente en la comprensión de la relación entre las características sanguíneas y la adaptación de los peces al ambiente (Tavares-Días y Moraes, 2004).

De los parámetros bioquímicos evaluados, solo la glucosa obtuvo valores dentro de los rangos reportados para reproductores y juveniles de B. amazonicus (Arias et al., 2003; Tavares-Días et al., 1999). Este parámetro bioquímico es un buen indicador de situaciones de estrés en peces (Sánchez et al., 2017). Por otro lado, la proteína plasmática evidenció resultados por debajo a lo reportado para reproductores (Arias et al., 2003). Al parecer, los valores de proteína pueden verse afectados por cambios en el volumen del plasma que puede ocurrir a causa del estrés u otros factores (Groff y Zinkl, 1999). Así mismo, existe referencia de correlación significativa positiva entre la proteína y el peso en hembras de Carassius 
auratus (Summerfelt et al., 1967); sin embargo, este comportamiento no se pudo determinar en el presente estudio dado que no se hizo la separación entre sexos. Los valores de los parámetros bioquímicos tienen una estrecha relación con el tipo de alimentación proporcionada a los peces durante el cultivo (Groff y Zinkl, 1999).

La concentración de leucocitos fue superior a lo reportado por Arias et al. (2003) y Tavares-Días et al. (1999), pero inferior al valor obtenido por Tavares-Días et al. (2008), en todos los casos en B. amazonicus. Los linfocitos son las células más abundantes y los valores encontrados coinciden con otros estudios de B. amazonicus (Arias et al., 2003; Tavares-Días et al., 1999, 2008), siendo estas células leucocitarias las que mayor porcentaje se presentan en diversas especies de teleósteos (Tavares-Días y Moraes, 2004; Gonzáles et al., 2016). Estas células cumplen un rol importante en el sistema inmune de los peces, actuando ante la presencia de virus, bacterias y otros agentes patogénicos que podrían comprometer la sobrevivencia del pez (Ruiz et al., 2003), y son sensibles a la presencia de contaminantes, cambios físicos y químicos del agua (Salazar-Lugo et al., 2012).

Los monocitos, al igual que los linfocitos, producen anticuerpos durante la respuesta inmune del pez (Tavares-Días et al., 2008) y en el presente estudio fueron la segunda célula de mayor abundancia, coincidiendo con lo reportado para Pterophyllum scalare (Iannacone et al., 2006). Sin embargo, los valores de los monocitos en otros reportes en B. amazonicus son bastante inferiores, donde la segunda célula sanguínea más abundante son los neutrófilos (Arias et al., 2003; Tavares-Días et al., 1999, 2008). Los eosinófilos estuvieron dentro del rango reportado para $B$. amazonicus y B. hilarii (Arias et al., 2003; Tavares-Días et al., 2003). Los eosinófilos se encuentren generalmente ausentes en peces (Tavares-Días y Moraes,
2004), donde altos porcentajes están relacionados a posibles infecciones parasitarias en Piaractus mesopotamicus y Colossoma macropomum (Ranzani-Paiva et al., 1999). No se reportó presencia de basófilos en Brycon, los cuales raramente se les encuentra en peces (Tavares-Días y De Moraes et al., 2004), pudiendo esta ausencia estar atribuida al uso de técnicas de coloración inadecuadas (López-Ruiz et al., 1992).

Las variaciones en la proporción de las células sanguíneas se pueden atribuir a la diversidad de técnicas de coloración para la identificación y cuantificación morfológica de los leucocitos; además de la dificultad en diferenciar monocitos de linfocitos, así como en la identificación de cada tipo de granulocito (Tavares-Días y De Moraes, 2004).

Las correlaciones positivas entre las variables hematológicas han sido reportadas en estudios de teleósteos (Bittencourt et al., 2003; Alesso et al., 2005; Macias y Rincon, 2010; Hahn-von-Hessberg et al., 2014). La hemoglobina, hematocrito, eritrocitos y los parámetros hematimétricos (VCM, HCM y CHCM) están íntimamente ligados, de allí que al modificarse alguno, los demás valores también pueden modificarse (Hahn-vonHessberg et al., 2014). Las correlaciones negativas encontradas en el presente estudio concuerdan con estudios realizados en Brycon henni, Leporinus obtusidens y Oreochomis niloticus (Alesso et al., 2005; Bittencourt et al., 2003; Macías y Rincón, 2010).

\section{Conclusiones}

Los valores hematológicos obtenidos en reproductores $B$. amazonicus en cautiverio muestran algunas variaciones con respecto a otras especies del género Brycon, pero se encuentran dentro del rango de otros teleósteos. 


\section{Agradecimientos}

Los autores manifiestan su agradecimiento a la Blga. Miriam Alvan Aguilar y al Blgo. Pedro Ramírez Arrarte (IAAP) por el apoyo durante la obtención de los peces y muestras de sangre. Al Dr. Marcos Tavares Días, responsable del Laboratorio de Patología y Sanidad de Organismos Acuáticos del EMBRAPA (Brasil), por sus valiosos aportes, revisiones y sugerencias del documento.

\section{Literatura Citada}

1. Alesso ME, Parma JM, Loteste A. 2005. Caracterización hematológica y bioquímica de Leporinus obtusidens (Val., 1847) (Pisces-Anostomidae). Natura Neotropicalis 36: 13-19. doi: 10.14409/natura.v1i36.3818

2. Arias JA, Benavides M, Hernández G, Eslava PR. 2003. Valoración hematológica y química sanguínea del yamú Brycon siebenthalae, en tres etapas de cultivo. Orinoquia 7: 34-41.

3. Bittencourt NLR, Molinari LM, Scoaris DO, Pedroso RB, Nakamura CV, Ueda-Nakamura T, Dias Filho BP. 2003. Haematological and biochemical values for Nile tilapia Oreochromis niloticus cultured in semiintensive system. Acta Scient 25: 385-389.

4. Blanco-Parra M, Bejarano-Rodríguez I. 2006. Diet and reproduction of the main fish species from the Mesay river (Colombian Amazon region) during the flooding season. Rev Biol Trop 54: 853-859.

5. Carneiro PC, Urbinati EC. 2001. Salt as a stress response mitigator of matrinxã, Brycon cephalus (Günther), during transport. Aquac Res 32: 297-304. doi: 10.1046/j.1365-2109.2001.00558.x

6. Carneiro PC, Urbinati EC. 2002. Ionic imbalance in matrinxã, Brycon cephalus (Teleostei: Characidae), submitted to different hauling densities. Aquacult Int 10:221-229.
7. Carneiro PC, Urbinati EC, Martins ML. 2002. Transport with different benzocaine concentrations and its consequences on hematological parameters and gill parasite populations of matrinxa Brycon cephalus (Gunther, 1869) (Osteichthyes, Characidae). Acta Scientiarum 24: 555-560.

8. Coffigny RS, Perez MM. 2003. Aceite de clavo (Syzygium aromaticum) como anestesico para la manipulação e transporte de Oreochromis aureus (Tilapia). [Internet]. Disponible en: http:// www.juntadeandalucia.es/agriculturaypesca/pesca/acuicultura/descargas/ NuevasEspecies/20_aceite_clavo_anestesico.pdf

9. García-Vásquez AR, Vargas G, TelloMartín JS, Duponchelle F. 2012. Desembarque de pescado fresco en la ciudad de Iquitos, región Loreto Amazonía peruana. Folia Amazónica 21: 45-52. doi: 10.24841/fa.v21i1-2.31

10. Goldenfarb B, Bowyer P, Hall E, Brosious E. 1971. Reproducibility in the hematology laboratory: the microhematocrit determination. Am J Clin Pathol 56: 35-39. doi: 10.1093/ajcp/56.1.35

11. Gonzáles A, Mejía F, Huanuiri K, Sánchez I, Vásquez J, FernándezMéndez C. 2016. Valores hematológicos y bioquímicos de juveniles de paiche Arapaima gigas en cultivo intensivo. Folia Amazónica 25: 137-144. doi: 10.24841/fa.v25i2.397

12. Groff JM, Zinkl JG. 1999. Hematology and clinical chemistry of cyprinid fish: common carp and goldfish. Vet Clin North Am Exot Anim Pract 2: 741-776. doi: 10.1016/S1094-9194(17)30120-2

13. Hahn-von-Hessberg CM, QuirozBucheli A, Grajales-Quintero A. 2014. Caracteres hematológicos en individuos de tilapia nilotica (Oreochromis niloticus, Trewavas 1983) con pesos entre 50-150 g y 150-250 g, estación piscícola, Universidad de Caldas, Colombia. Bol Cient Mus Hist Nat 18: 142-157. 
14. Hawkey M, Dennett B. 1989. Color atlas of comparative veterinary hematology. Vet Clin Path 18: 108-108. doi: 10.1111/j.1939-165X.1989.tb00532.x

15. Iannacone J, Bello C, Hernández N, Díaz M. 2006. Caracterización leucocitaria del pez amazónico Pterophyllum scalare (Lichtenstein, 1823) (Perciformes: Cichlidae) de Perú. Biotempo 6: 3237. doi: 10.31381/biotempo.v6i0.880

16. Larsson A, Johansson Sjöbeck MJ, Fänge R. 1976. Comparative study of some haematological and biochemical blood parameters in fishes from the Skagerrak. J Fish Biol 9: 425-440. doi: 10.1111/j.1095-8649.1976.tb04691.x

17. Leonardo AF, Hoshiba MA, Senhorini JA, Urbinati EC. 2008. Canibalismo em larvas de matrinxã, Brycon cephalus, após imersão dos ovos à diferentes concentrações de triiodotironina (T3). Bol Inst Pesca 34: 231-239.

18. Lima FC. 2017. A review of the cisandean species of the genus Brycon Müller y Troschel (Characiformes: Characidae). Zootaxa 4222: 1-189. doi: 10.11646/zootaxa.4222.1.1

19. López-Ruiz A, Angeles-Esteban M, Meseguer J. 1992. Blood cell of the gilthead seabream (Sparus aurata L.): Ligth and electron microscopic studies. Anat Rec 234: 161-171. doi: 10.1002/ ar. 1092340203

20. Macías JL, Rincón ER. 2010. Caracterización sanguínea de diferentes especies ícticas de la cuenca alta del rio Cauca (Colombia). Acta Biol Paranaense 39: 71-86.

21. Rocha RM, Carvalho EG, Urbinati EC. 2004. Physiological responses associated with capture and crowding stress in matrinxã Brycon cephalus (Gunther, 1869). Aquac Res 35: 245-249. doi: 10.1111/j.1365-2109.2004.00998.x

22. McDonald G, Milligan CL. 1997. Ionic, osmotic and acid-base regulation in stress. In: Fish stress and health in aquaculture. Cambridge, UK: Cambridge University Press. p 119-145.
23. Natt P, Herrick A. 1952. A new blood diluent for counting the erythrocytes and leucocytes of the chicken. Poultry Sci 31: 735-738. doi: 10.3382/ps.0310735

24. Nikinmaa M. 1990. Vertebrate red blood cells. Adaptations of function to respiratory requirements. Berlin: Springer-Verlag. $262 \mathrm{p}$.

25. Nuñez J. 2009. Domestication de nouvelles espèces d'intérêt piscicole en Amazonie. Cah Agric 18: 136-143. doi: 10.1684/agr.2009.0299

26. Oliveira AT, Pantoja-Lima J, Aride, PHR, Tavares-Dias M, Marcon JL. 2015. Fisiologia de arraias de água doce: subsídios para aplicabilidade na aquicultura. En: Tavares-Dias M, Mariano W (eds). Aquicultura no Brasil: novas perspectivas. Brasil: Pedro y João Editores. p 45-74.

27. Pizango-Paima EG, Pereira-Filho M, De Oliveira-Pereira MI. 2001. Body composition and food of the matrinxã, Brycon cephalus (Günther, 1869) (Characiformes, Characidae) from central Amazonia. Acta Amazon 31: 509509. doi: 10.1590/1809-43922001313520

28. Paiva MJ. 1991. Características sanguíneas da Pirapitinga do Sul, Brycon sp, sob condições experimentais de criação intensiva. Braz J Vet Res Anim Sci 28: 141-153. doi: 10.11606/issn.16784456.bjvras.1991.51933

29. Ranzani-Paiva MJ, Salles FA, Eiras JC, Eiras AC, Ishikawa CM, Alexandrino $A C$. 1999. Análises hematológicas de curimbatá (Prochilodus scrofa), pacu (Piaractus mesopotamicus) e tambaqui (Colossoma macropomum) das estações de piscicultura do Instituto de Pesca, Estado de São Paulo. Bol Inst Pesca 25: 77-83.

30. Ranzani-Paiva MJ, Pádua SB, Tavares-Dias M, Egami M. 2013. Métodos para análise hematológica em peixes. Maringá, Paraná: Eduem. 140 p.

31. Romagosa E, Narahara MY, Borella MI, Fenerich-Verani N. 2001. Seleção e caracterização de fêmeas de matrinxã, Brycon cephalus induzidas a reprodução. Bol Inst Pesca 27: 139-147. 
32. Ruiz I, Fernández AB, De Blas I. 2003. El sistema inmune de los teleósteos (III): Respuesta inmune específica. AquaTIC 18: 33-38.

33. Salazar-Lugo R, Romero Z, Centeno L. 2012. Caracterización morfológica y citoquímica de leucocitos del pez dulceacuícola Colossoma macropomum (Characiformes: Characidae). Saber 24: 49-55.

34. Sánchez I, Mejía F, Huanuiri K, Vásquez J, Gonzales A, FernándezMéndez C. 2017. Respuesta hematológica y bioquímica en juveniles de paiche Arapaima gigas sometidos a diferentes concentraciones de amonio. Folia Amazónica 26: 51-58. doi: 10.24841/ fa.v26i1.418

35. Summerfelt RC, Lewis WM, Ulrich MG. 1967. Measurement of some hematological characteristics of the goldfish. Prog Fish Cult 29: 13-20. doi: $10.1577 / 1548-8640$ (1967) 29[13:MOSH-CO]2.0.CO;2

36. Tandon RS, Joshi BD. 1976. Total red and white blood cell count of 33 species of fresh water teleosts. J Anim Physiol An N 37: 293-297. doi: 10.1111/j.14390396.1976.tb00069.x

37. Tavares-Dias M, Frascá-Scorvo CM, Moraes FR, Campos-Filho E. 1999. Características hematológicas de teleósteos brasileiros. IV. Parâmetros eritroleucométricos, trombométricos e glicemia do matrinxã (Brycon cephalus Günther, 1869) (Osteichthyes: Characidae). Rev Bras Zool 15: 149-153.

38. Tavares-Dias M, Schalch SE, Moraes FR. 2003. Hematological characteristics of Brazilian teleosts. VII. Parameters of seven species collected in Guariba, São Paulo State, Brazil. Bol Inst Pesca 29: 109-115.
39. Tavares-Días M, De Moraes FR. 2004. Hematologia de peixes teleósteos. Ribeirão Preto: Villimpress. $144 \mathrm{p}$.

40. Tavares-Dias M, Moraes FR. 2006. Hematological parameters for the Brycon orbignyanus Valenciennes, 1850 (Osteichthyes: Characidae) intensively bred. Hidrobiologica 16: 271-274.

41. Tavares-Días M, Affonso EG, Oliveira SR, Marcon JL, Egami MI. 2008. Comparative study on hematological parameters of farmed matrinxã, Brycon amazonicus Spix and Agassiz, 1829 (Characidae: Bryconinae) with others Bryconinae species. Acta Amazon 38: 799-805. doi: 10.1590/S004459672008000400026

42. Urbinati EC, Carneiro PC. 2001. Metabolic and hormonal responses of matrinxa, Brycon cephalus (Teleost: Characidae) to transport stress under influence benzocaine. J Aquacul Tropics 16: 75-85.

43. Urbinati EC, de Abreu JS, da Silva CA, Landinez PM. 2004. Loading and transport stress of juvenile matrinxa (Brycon cephalus, Characidae) at various densities. Aquaculture 229: 389-400. doi: 10.1016/S0044-8486(03)00350-8

44. Val AL, de Almeida-Val VM. 1995. Fishes of the Amazon and their environment: Physiological and biochemical aspect. Berlin, Germany: SpringerVerlag. $224 \mathrm{p}$.

45. Wintrobe M. 1934. Variations on the size and haemoglobin content of erythrocytes in the blood various vertebrates. Folia Haematologica 51: 32-49.

46. Zaniboni-Filho E, Reynalte-Tataje D, Weingartner M. 2006. Potencialidad del género Brycon en la piscicultura brasileña. Rev Colomb Cienc Pec 19: 233240. 